\title{
MODELO 3D PLAZA DE LAS ESTELAS COPÁN: ANÁLISIS ARQUEOASTRONÓMICO PRELIMINAR
}

\author{
Javier Mejuto-González ${ }^{1, *}$ Eduardo Rodas-Quito ${ }^{1,+}$ \\ ${ }^{1}$ Departamento de Arqueoastronomía y Astronomía Cultural, UNAH \\ Recibido: 03/junio/2019 Aceptado: 10/octubre/2019 \\ DOI: https://doi.org/10.5377/ce.v12i1.9629
}

\begin{abstract}
RESUMEN
En este trabajo se presenta el modelo 3D de la plaza de las estelas del sitio arqueológico de Copan. Este se realizó principalmente con tres objetivos: conocer la motivación para construir dichos monumentos, entender la importancia de algunas fechas en el calendario maya al relacionar las decoraciones e inscripciones con las fechas en que ocurren los fenómenos solares según la posición de los monumentos analizados y el tipo de relación que los gobernantes creyeron tener entre ellos y las diferentes deidades en el panteón maya, especialmente las relacionadas con el dios solar, K'inich Ajaw. La conclusión de este estudio es que parece haber una relación entre las estelas A-H y A-I de carácter astronómico enraizado en la cosmovisión maya con la finalidad de justificar el poder dinástico real en Copán ruinas.
\end{abstract}

Palabras clave: Astronomía cultural, arqueoastronomía, Ajaw, Copán ruinas.

\begin{abstract}
In this work a 3D model for the stela plaza of the archaeological site of Copan is presented. This model will help understanding the motivation behind the stelas as well as understanding the relevance of some dates in the Mayan calendar by relating the decorations and inscriptions with solar events and the relationship between the rulers and other divine entities (especially those related to the solar god, K'inich Ajaw). According to this study it appears an astronomical relationship between stelae A-H and A-I probably as an attempt to justify the royal power in Copan.
\end{abstract}

Keywords: Cultural astronomy, archaeoastronomy, Ajaw, Copan ruinas.

\section{Introducción}

Los estudios arqueoastronómicos, como cualquier otro estudio de tipo geoespacial, requieren de una serie de recursos para la toma de datos en el campo. Sin embargo, en los últimos tiempos es posible reducir costes -aunque no eliminarlos- a través del uso de modelos digitales que permiten el estudio de ciertos elementos sin el desplazamiento físico al lugar de estudio. Parece obvio comentar que estos estudios y los resultados relacionados con el mismo serán tan relevantes como lo sea el modelo que se obtenga. Por

*javier.mejuto@unah.edu.hn, ORCID: https:/ / orcid.org/0000-0001-8936-7236

†eduardo.rodas@unah.edu.hn, ORCID: https:/ /orcid.org/0000-0001-6956-0987 
tanto, en la elaboración de dichos modelos se deben controlar de manera muy especial los factores que afectan la precisión de los resultados derivados del uso de estos modelos. Las dimensiones del sitio en estudio, su estado de conservación y las mismas herramientas para la creación y manipulación de modelos son algunos de las variables a controlar por parte del investigador. Como consecuencia de ello, además de la comprensión de la motivación de quienes construyeron estas estructuras antiguas, también permite indagar en los elementos intangibles que están asociados a dichos restos y que son del interés no solo de los estudiosos de las antiguas sociedades sino que de las comunidades en que se encuentran, dado el potencial turístico y económico que dicho interés provoca en la sociedad en general. Por otro lado, este estudio requiere, al menos en el caso de la Arqueoastronomía, la observación de ciertos fenómenos celestes, los que no son frecuentes sino que suceden en épocas específicas del año, por tanto el tiempo es un factor que limita la realización de pruebas de verificación de las hipótesis planteadas por los arqueoastrónomos y surge la necesidad de crear modelos digitales que permitan acortar el tiempo invertido en su estudio y la reducción y falsación de hipótesis.

En el caso de las Ruinas de Copán, como en una gran mayoría de sitios arqueológicos, los monumentos están en constante riesgo de destrucción debido tanto a procesos naturales de erosión como factores antropogénicos. Este hecho tiene que ser tomado en cuenta antes de proponer cualquier desarrollo que puede agravar este problema (CATIE, 1984).

Estudios arqueoastronómicos han evidenciado que el sector norte de la Plaza Principal del Parque Arqueológico de Copán Ruinas, denominado Plaza del Sol, fue construido por los Mayas para marcar alineamientos astronómicos del Sol (Pineda de Carías et al., 2009). Sin embargo, no están fácilmente disponibles modelos tridimensionales (3D) y de realidad virtual que permitan estudiar con más profundidad estos alineamientos. Tampoco se cuenta con bases de datos y sistemas de información geográfica que utilicen tecnologías de vanguardia para estudios arqueoastronómicos más extensos.

\section{Metodología}

La metodología empleada consistió en el modelado tridimensional del sector norte de la plaza principal a partir de los datos obtenidos de los mapas ya realizados en las diferentes investigaciones llevadas a cabo en el Parque Arqueológico de Copán Ruinas (PACR) y con el modelo desarrollado, realizar el análisis y simulación de las luces y sombras generadas por la interacción de la iluminación solar con las estelas, las que podrían coincidir en el tiempo con eventos importantes del ciclo solar anual, que se corresponden, a su vez, con eventos de relevancia en la vida social de los antiguos habitantes mayas del lugar.

Aunque ya existen actualmente otras herramientas que permiten realizar análisis virtuales del PACR, tales como el proyecto MayaArch3D ( www.mayaarch3d.org; Schwerin et al., 2011), éstos se circunscriben al análisis desde el punto de vista antropológico de restos materiales, es decir, aspectos tales como el estilo de las esculturas, estilo de decoraciones de monumentos, cerámicas o de escritura, entre otros así como del punto de vista patrimonial, al preservar digitalmente un modelo virtual de los artefactos encontrados en el PACR para posteriores análisis, sin generar daño ni desgaste al artefacto original por la necesaria exposición a los elementos naturales y a los instrumentos de análisis. Por otro lado, estos modelos existentes fueron diseñados para el análisis individual de los artefactos, no para estudiar las interacciones que tienen entre sí o con el medio que les rodea. Sin embargo, si quien investiga tiene habilidades de tipo informático, es posible hacer una transferencia de estos modelos de alta calidad a software de tipo astronómico, como Stellarium (Zotti et al., 2017) y a partir de allí identificar las relaciones entre fenómenos astronómicos y orientaciones de estructuras en sitios arqueológicos. Dado que el estudio descrito en el presente trabajo 
es de tipo preliminar, no es necesario manejar la gran cantidad de datos que hay que transferir desde las bases de datos de los sitios descritos en este párrafo, por lo que se optó por buscar alternativas más sencillas que se pudieran utilizar con los datos con que ya se cuenta del sitio bajo estudio.

Por tanto fue necesario utilizar una herramienta que permitiera incorporar diversas formas de análisis de los modelos 3D existentes, desde las posiciones relativas de cada artefacto que permitiera un análisis de sus interacciones, hasta la forma cómo la iluminación solar crea patrones en las sombras de los mismos. Se encontró que para el presente análisis, el software "SketchUp" en su versión 8, www.sketchup.com, cumplía con los requerimientos necesarios, ya que es un software gratuito y con el mismo se pueden crear modelos 3D de estructuras, dándoles una forma muy aproximada al real, los que se pueden georreferenciar ingresando las coordenadas de un punto específico del modelo y de esta manera, simular la iluminación del Sol y por tanto las sombras generadas por las estructuras de interés. Estas sombras, a su vez, permitieron encontrar patrones que se pueden relacionar con las fechas de eventos que pudieron haber sido importantes en la sociedad de los mayas antiguos. De esta forma, se puede:

i. Conocer la motivación para construir dichos monumentos

ii. Entender la importancia de algunas fechas en el calendario maya al relacionar las decoraciones e inscripciones con las fechas en que ocurren los fenómenos solares según la posición de los monumentos analizados

iii. El tipo de relación que los gobernantes creyeron tener entre ellos y las diferentes deidades en el panteón maya, especialmente las relacionadas con el dios solar, K'in.

Los modelos digitales permiten realizar estudios muy cercanos a la realidad, volviendo innecesaria la exposición de los artefactos originales a los correspondientes riesgos inherentes del estudio. Para obtener resultados realistas, es muy importante que al elaborar los modelos digitales, se tomen los datos más detallados y precisos a partir de los originales. Para esto, se procedió como sigue:

i. Se tomaron medidas físicas directamente de los originales

ii. Se tomaron datos de diversas fuentes documentales para corroborar los datos ya recolectados así como para obtener otros con que todavía no se contaba

iii. Con estos datos, se procedió a su organización y clasificación en una base de datos digital, por monumento estudiado

iv. Finalmente, se definió el área de estudio sobre un mapa del área de interés, en este caso, la Plaza Principal.

Con estos datos ya organizados y clasificados, se elaboraron los modelos digitales con la herramienta SketchUp. Se corrieron simulaciones de sombras de estructuras en diversas fechas, buscando aquellas en que coincidieran los extremos de las sombras con la base o esquina de otro monumento, agregándose estas fechas en la base de datos, por monumento que la generaba, a lo largo de un período correspondiente a un año trópico. También se registró el monumento con el que coincidía cada uno de ellos en esta relación de luz y sombras. 


\section{Resultados}

Se construyó un modelo 3D virtual del sector Norte de la Plaza Principal de Copán Ruinas, utilizando las siguientes fuentes para los datos del modelo:

1) Google Earth, gracias a la capacidad de SketchUp de georreferenciar estructuras elaboradas con este software, utilizando este servicio web de información geográfica

2) Datos de campo obtenidos por docentes del Departamento de Arqueoastronomía y Astronomía Cultural (dimensiones de las estelas).

Con estos datos, se procedió a generar modelos virtuales de estelas y escalinata oeste de la Plaza Principal, utilizando para ello el software SketchUp, que permite la creación de modelos de este tipo de forma realista, a partir de las dimensiones que el usuario ingrese. Los modelos para cada estela y la estela se ubicaron en las posiciones geográficas que les corresponden gracias a la capacidad de SketchUp de descargar del servicio Google Earth la fotografía del sitio geográfico que especifique el usuario y sobre él, ubicar los modelos virtuales generados. Luego, se georreferenció el mapa descargado a las coordenadas correspondientes. En las figuras 1, 2, 3 y 4 se presentan capturas de pantalla del modelo elaborado.

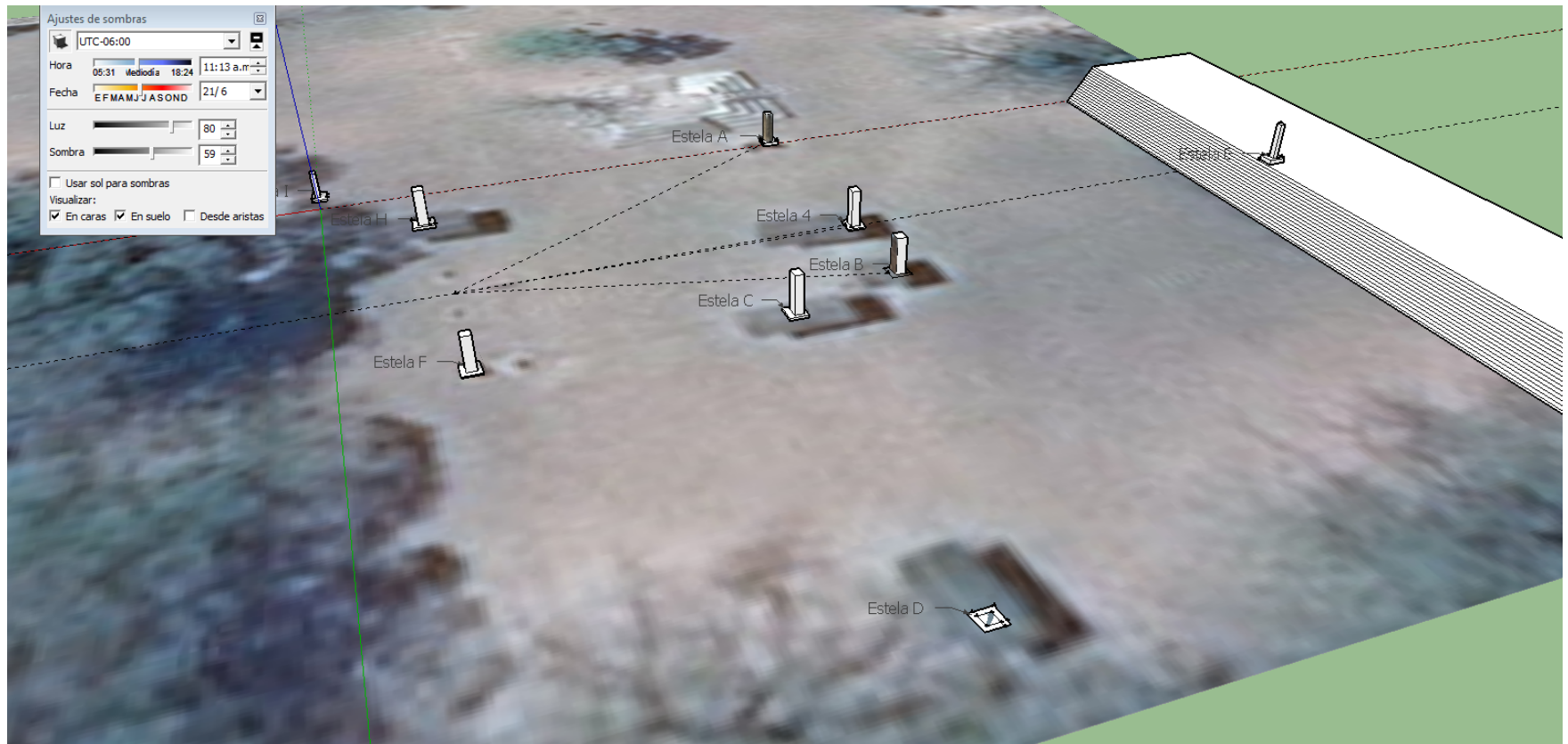

Figura 1: Vista desde el norte del modelo virtual de la Gran Plaza de Copán. 


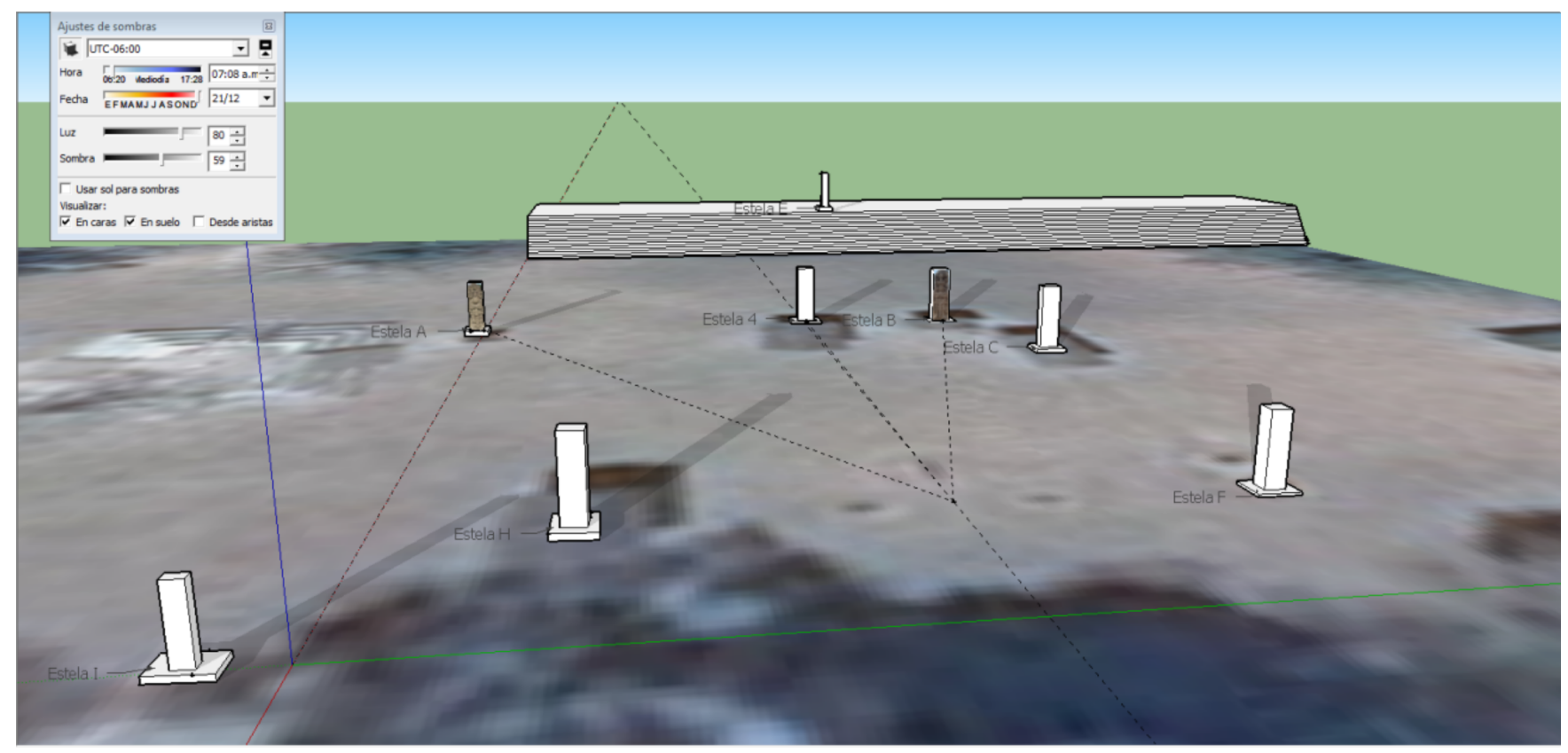

Figura 2: Vista desde el este del modelo virtual de la Gran Plaza de Copán

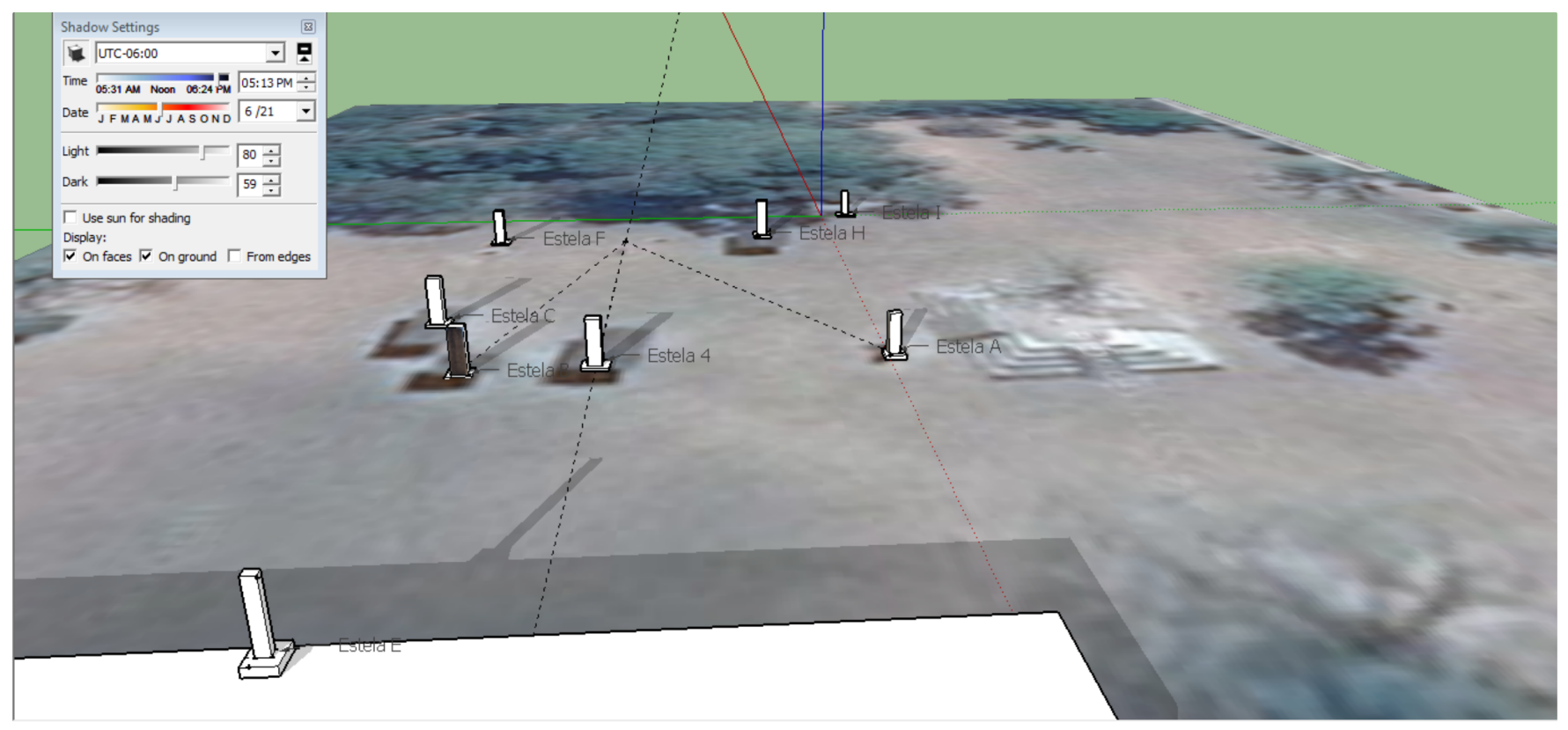

Figura 3: Vista desde el oeste del modelo virtual de la Gran Plaza de Copán. 


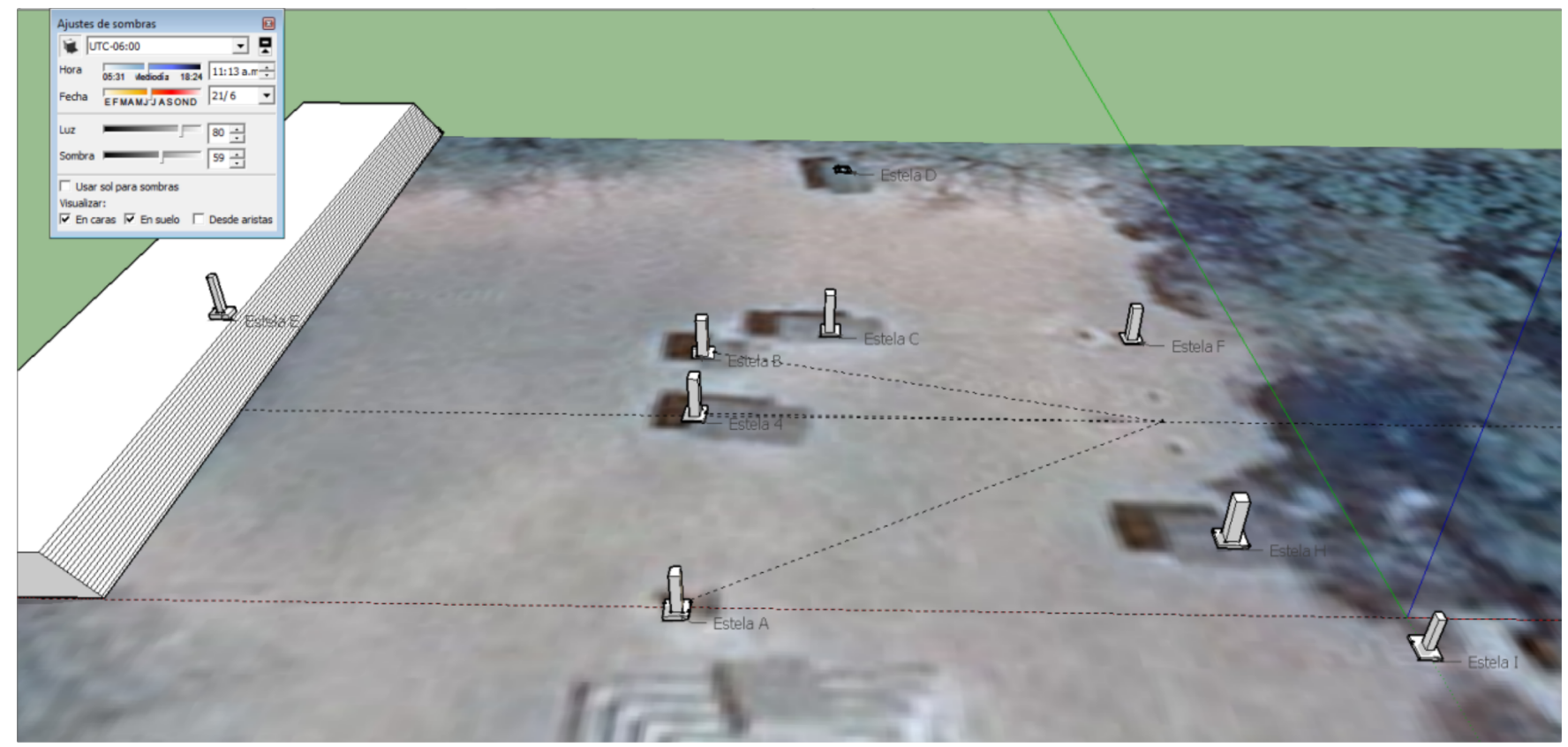

Figura 4: Vista desde el sur del modelo virtual de la Gran Plaza de Copán.

El siguiente paso fue utilizar la capacidad de SketchUp de simular la posición del Sol según la hora del día y la época del año. De esta manera se identificaron las fechas en que coinciden las sombras proyectadas por cada estela sobre las otras de esta misma Plaza, identificándose alineaciones en varias fechas, las que se muestran en la tabla 1.

Tabla 1: Posibles alineaciones entre estelas de la Gran Plaza de Copán.

\begin{tabular}{ccccc}
\hline$N^{\circ}$. Alineación & $\begin{array}{c}\text { Fechas que ocurre } \\
(\mathrm{dd} / \mathrm{mm}-\mathrm{dd} / \mathrm{mm})\end{array}$ & $\begin{array}{c}(\mathrm{A}) \text { manecer } /(\mathrm{O}) \text { caso } \\
\text { Estela proyectando } \\
\text { la sombra }\end{array}$ & $\begin{array}{c}\text { Estela recibiendo } \\
\text { sombra }\end{array}$ \\
\hline 1 & $29 / 01-12 / 11$ & $\mathrm{~A}$ & $\mathrm{H}$ & 4 \\
2 & $17 / 03-26 / 09$ & $\mathrm{~A}$ & $\mathrm{I}$ & $\mathrm{A}$ \\
3 & $21 / 03-21 / 09$ & $\mathrm{~A}$ & $\mathrm{~F}$ & $\mathrm{C}$ \\
4 & $06 / 04-07 / 09$ & $\mathrm{~A}$ & $\mathrm{~F}$ & $\mathrm{~B}$ \\
5 & $06 / 04-08 / 09$ & $\mathrm{~A}$ & $\mathrm{I}$ & Esquina NE est.4 \\
6 & $25 / 04-19 / 08$ & $\mathrm{~A}$ & $\mathrm{H}$ & $\mathrm{A}$ \\
7 & $17 / 05-25 / 07$ & $\mathrm{~A}$ & $\mathrm{~F}$ & 4 \\
8 & $01 / 02-10 / 11$ & $\mathrm{O}$ & 4 & $\mathrm{~F}$ \\
9 & $09 / 02-1 / 11$ & $\mathrm{O}$ & $\mathrm{B}$ & $\mathrm{C}$ \\
10 & $21 / 02-20 / 10$ & $\mathrm{O}$ & $\mathrm{A}$ & $\mathrm{H}$ \\
11 & $11 / 03-02 / 10$ & $\mathrm{O}$ & $\mathrm{E}$ & $\mathrm{C}$ \\
12 & $11 / 03-02 / 10$ & $\mathrm{O}$ & $\mathrm{B}$ & $\mathrm{F}$ \\
13 & $15 / 03-27 / 09$ & $\mathrm{O}$ & $\mathrm{E}$ & $\mathrm{B}$ \\
14 & $22 / 03-21 / 09$ & $\mathrm{O}$ & $\mathrm{E}$ & $\mathrm{I}$ \\
15 & $28 / 03-14 / 09$ & $\mathrm{O}$ & $\mathrm{A}$ & $\mathrm{F}$ \\
16 & $28 / 03-14 / 09$ & $\mathrm{O}$ & $\mathrm{C}$ & 4 \\
17 & $25 / 04-14 / 09$ & $\mathrm{O}$ & $\mathrm{E}$ & $\mathrm{H}$ \\
18 & $05 / 05-07 / 08$ & $\mathrm{O}$ & 4 & $\mathrm{H}$ \\
19 & $21 / 05-22 / 07$ & $\mathrm{O}$ & $\mathrm{A}$ & $\mathrm{I}$ \\
20 & $21 / 05-22 / 07$ & $\mathrm{O}$ & $\mathrm{C}$ & \\
\hline
\end{tabular}




\section{Discusión y resultados}

Tras el análisis de las sombras se identificaron un total de 20 posibles alineaciones de tipo solar entre las estelas de la Plaza Principal. Sin embargo, tras el análisis astronómico y cultural respectivo se descartaron las alineaciones 1 y 2 ya que para que se den es necesario que el Sol se encuentre en un punto por debajo del horizonte local, por tanto, no es posible que ocurran dichas alineaciones. Dada la gran cantidad de alineaciones restantes, se concentró el estudio en aquellos alineamientos cuyas fechas tengan un especial significado astronómico. Por lo tanto, se analizan las orientaciones número 3 (equinoccio de primavera), 4, 5 (dado que ocurren en la misma fecha) y 6 (día del paso del Sol por el cenit) en el orto solar y los alineamientos 14 (equinoccio de primavera), 15 y 16 (dado que ocurren en la misma fecha) y 17 (día del paso del Sol por el cenit) para el ocaso solar. Los datos de estas alineaciones se muestran en la tabla 2.

Tabla 2: Posibles alineaciones entre estelas de la Gran Plaza de Copán

\begin{tabular}{ccccc}
\hline $\mathrm{N}^{\circ}$. Alineación & $\begin{array}{c}\text { Fechas que ocurre } \\
(\mathrm{dd} / \mathrm{mm}-\mathrm{dd} / \mathrm{mm})\end{array}$ & $\begin{array}{c}(\mathrm{A}) \text { manecer/(O)caso } \\
\text { Estela proyectando } \\
\text { la sombra }\end{array}$ & $\begin{array}{c}\text { Estela recibiendo } \\
\text { sombra }\end{array}$ \\
\hline 3 & $21 / 03-21 / 09$ & $\mathrm{~A}$ & $\mathrm{~F}$ & $\mathrm{C}$ \\
4 & $06 / 04-07 / 09$ & $\mathrm{~A}$ & $\mathrm{~F}$ & $\mathrm{~B}$ \\
5 & $06 / 04-08 / 09$ & $\mathrm{~A}$ & $\mathrm{I}$ & Esquina NE est.4 \\
6 & $25 / 04-19 / 08$ & $\mathrm{~A}$ & $\mathrm{H}$ & $\mathrm{A}$ \\
14 & $22 / 03-21 / 09$ & $\mathrm{O}$ & $\mathrm{E}$ & $\mathrm{B}$ \\
15 & $28 / 03-14 / 09$ & $\mathrm{O}$ & $\mathrm{A}$ & $\mathrm{I}$ \\
16 & $28 / 03-14 / 09$ & $\mathrm{O}$ & $\mathrm{C}$ & $\mathrm{F}$ \\
17 & $25 / 04-14 / 08$ & $\mathrm{O}$ & $\mathrm{E}$ & 4 \\
\hline
\end{tabular}

Para la mayoría de los alineamientos parece existir cierto patrón interpretativo en el que a través de un sacrificio de sangre se renueva el universo y se asegura la prosperidad del sitio de Copán y sus habitantes, realizándose -a juzgar por las relaciones que infieren las sombras de estelas entre ellas- en el orto u ocaso solar de los equinoccios o fechas cercanas, así ocurre con las alineaciones 4 y 14. Con el mismo tema de renovación cósmica aparecen las alineaciones 5 y 17 pero en este caso son las dos únicas estelas que respetó Waxaklajun Ubah Kawiil previas a su mandato, ambas de su abuelo K'ahk' U Ti' Chan Yopaat y ambas proyectan la sombra sobre la estructura 4 que amerita mayores investigaciones ya que esta estructura, a falta de una datación concluyente, se supone posterior a este período. Por último, las alineaciones 3 y 16 son de tema cosmogónico pero ninguna parece tener una explicación astronómica en la base y podría ser meras coincidencias como en los casos desechados previamente.

Fijemos la atención ahora en los alineamientos 6 y 15. El alineamiento 6 se produce en el orto del paso del sol por el cénit -tanto el primero como el segundo- proyectando la estela H su sombra sobre la estela A. La estela A representa al decimotercer gobernante de Copán Waxaklajun Ubah Kawiil, como dios patrono de Copán y hace referencia al ciclo de muerte, resurrección y apoteosis para honrar a su abuelo K'ahk' U Ti' Chan Yopaat que gobernó el sitio entre los años 578 y 628 d.C. En la estela H el mismo Waxaklajun Ubah Kawiil se hace representar como creador del cosmos en la figura del dios del Maíz, la fecha de advocación de esta estela 9.14.19.5.0 solamente difiere 260 días de la de la estela A, 9.15.0.0.0. Por lo tanto, están relacionadas (Agurcia Fasquelle y Véliz, 2010) con el ciclo sagrado de 260 días, Tzolkin y el período de visibilidad como estrella vespertina y lucero del alba de Venus. Es aceptado en la bibliografía el carácter agrícola del ciclo de 260 dias y así se traduce de la relación entre las estelas H y A. La estela H y Waxaklajun Ubah Kawiil como dios del Maíz proyecta su sombra sobre la estela A que posee motivos del ciclo de Muerte, Resurrección y Apoteosis. El dios de maíz, representando como el Ajaw proyecta esta 
sombra el día del paso del Sol por el cénit, día que marca el inicio de la época lluviosa en mesoamérica y que por lo tanto tiene un eminente protagonismo en la preparación anual de los campos de siembra.

En cuanto al alineamiento 15, involucra las estelas A e I. Como se ha comentado previamente la estela A representa al decimotercer gobernante de Copán Waxaklajun Ubah Kawiil honrando a su abuelo K'ahk' U Ti' Chan Yopaat.

Durante mucho tiempo la estela I ha llamado la atención por estar empotrada en la gradería Sur de la plaza principal -sin haber sido destruida por Waxaklajun Ubah Kawiil quien modificó esta plaza para colocar sus estructuras- por lo tanto cabe pensar que se trata de una estela de importancia para el decimotercer gobernante de la dinastía. Esta estela fue realizada por K'ahk' U Ti' Chan Yopaat haciendo referencia a tres de los dioses patronos de Copán: Chan Te' Ch'oktaak, B'alu'n Te'Witz y B'alu'n k'awiil. También aparece el gobernante mítico K'ihnich Yajaw Hu'n haciendo referencia al asentamiento del poder de la dinastía preponderante en Copán.

Teniendo en cuenta estas dos estelas y que es la A la que proyecta su sombra sobre la I durante los ocasos los días de los equinoccios podemos inferir que Waxaklajun Ubah Kawil pretendía conectarse con su pasado dinástico a través de su abuelo y el gobernante mítico K'ihnich Yajaw Hu'n. Asimismo se hace representar como un dios patrono de Copán aunándose a los 3 dioses patronos que aparecen en la estela I. Todo ello se hace coincidir con el ocaso en los equinoccios, donde el sol se oculta exactamente por el Oeste, representando la muerte de sus antepasados, en especial, la de su abuelo que lo conecta directamente con la dinastía gobernante de Copán y celebrando el ciclo Muerte-Resurrección-Apoteosis del Sol se identifica con el K'ihnich Ajaw, mediante este juego de sombras.

Por lo tanto, parece haber una relación entre las estelas A-H y A-I de carácter astronómico. En ambos casos el Ajaw utiliza elementos de la cosmovisión maya para apoyar su poder dinástico basado en elementos que incluyen la visión cultural maya de Venus y el Sol y sus respectivas divinidades con hierofanías que se expresan a través de sombras proyectadas en días específicos del año teniendo en cuenta el horizonte local.

\section{Referencias}

Agurcia Fasquelle, R. y Véliz, S. (2010). Manual de los monumento de copán. Obtenido de http: //famsi.org/research/copan/monuments/CopanMonumentManual.pdf, Copán, Honduras.

CATIE (1984). Plan de Manejo y Desarrollo del Monumento Nacional Ruinas de Copán. Turrialba, Costa Rica.

Pineda de Carías, M. C., Véliz, S. V., y Agurcia Fasquelle, R. (2009). Estela d: Reloj solar de la plaza del sol del parque arqueológico de copán ruinas, honduras. Yaxkin, 15:111-138.

Schwerin, J. v., Richards-Rissetto, H., Remondino, F., Agugiaro, G., Forte, M., y Maqueda, R. (2011). The mayaarch3d project: Digital technologies for research in maya archaeology. Technical report. Obtenido de http: / / mayaarch3d.org.

Zotti, G., Schaukowitsch, F., y Wimmer, M. (2017). Beyond 3d models: Simulation of temporally evolving models in stellarium. Mediterranean Archaeology and Archaeometry, 18(4):501-506. Obtenido de http: //maajournal.com/Issues/2018/Vol18-4/63_Zotti\%20et\%20al\%2018(4).pdf. 